\title{
Comportamento da parasitemia pelo Trypanosoma cruzi em chagásicos crônicos durante 13 anos
}

\author{
Aspects of parasitemia by Trypanosoma cruzi in chronic \\ chagasic patients during 13 years
}

Cleudson Castro, Vanize Macêdo e Aluízio Prata

\begin{abstract}
Resumo Foi estudada a parasitemia de 202 chagásicos crônicos por um período médio de 13 anos, através de repetidos xenodiagnósticos convencionais. Os pacientes tinham média de idade de 41,4 anos, residiam em área endêmica, sendo 124 do sexo feminino e 78 do sexo masculino. Foi observado que o nível de parasitemia aumentou em 14 indivíduos, diminuiu em 42 e permaneceu inalterado em 146. Porém, no geral, a parasitemia declinou. Os percentuais de chagásicos xenopositivos que foram $37,6 \%, 48,5 \%, 51 \%$ no primeiro, segundo e terceiro xenodiagnósticos, respectivamente, em 1976/78, passaram a ser 30,2\% em 1988/91 ( $p=0,00003$ ). Os percentuais de pools positivos que foram 15,2\%, 20,9\%, 20,8\% no primeiro, segundo e terceiro xenodiagnósticos, respectivamente, em 1976/78, passaram a ser 10,4\% em 1988/91, $(p=0.00000001)$. Houve 62 pacientes que tiveram todos os xenodiagnósticos negativos e 23 que apresentaram todos os exames positivos. Os percentuais de chagásicos com alta, média e baixa parasitemias que, em 1976/78, foram, respectivamente, 9,4\%; 20,8\% e 69,8\% passaram a ser, em 1988/91, respectivamente, 4,4\%, 12,9\% e 82,7\%.
\end{abstract}

Palavras-chaves: Parasitemia longitudinal. Parasitemia em chagásico crônico. Parasitemia persistente no chagásico.

\begin{abstract}
The parasitemia of 202 chronic chagasics was studied for approximately 13 years by repetead conventional xenodiagnoses. Mean patient age was 41.1 years. They lived in an endemic area; 124 were females and 78 were males. It was seen that the level of parasitemia oscillated. It went up in 14 individuals, went down in 42 and stayed at the same level in 146. In general the parasitemia was reduced. The percentage of xenopositive chagasics, which was $37.6 \%, 48.5 \%$, and $51 \%$ in the first, second and third xenodiagnosis, respectively, in 1976/78, changed to $30.2 \%$ in $1988 / 91$ ( $p=0.00003$ ). The percentage of positive pools, whicht was $15.2 \%, 20.9 \%, 20.8 \%$ in the first, second and third xenodiagnosis, respectively, in 1976/78, changed to $10.4 \%$ in 1988/91, $(p=0.00000001)$. There were 62 patients whose xenodiagnoses were all negative and 23 whose exams were all positive. The percentage of chagasics with high, medium and low parasitemia, which in 1976/78 was 9.4\%, 20.8\% and 69.8\%, respectively, changed to $4.4 \%, 12.9 \%$ and $82.7 \%$, respectively, in 1988/91.
\end{abstract}

Key-words: Longitudinal parasitemia. Parasitemia in chronic chagasics. Persistent parasitemia in chagasics.

Núcleo de Medicina Tropical e Nutrição, Universidade de Brasília, Brasília DF, Brasil.

Endereço para correspondência: Dr. Cleudson Castro. Núcleo de Medicina Tropical e Nutrição/UnB. Caixa Postal 4517, 70919-970 Brasília, DF, Brasil.

Fax: 5561 273-2811

Recebido para publicação em 6/5/98. 
Apesar do chagásico crônico apresentar xenodiagnóstico positivo durante muitos anos, mesmo após abandonar as áreas endêmicas 813 , a comprovação periódica e repetida da parasitemia dos pacientes tem sido demonstrada por poucos investigadores. Assim, Pereira et al14 15 estudaram a parasitemia em pacientes de Virgem da Lapa em duas ou mais oportunidades em um intervalo de dez anos. Cerisola e Rohwedder 5 acompanharam pacientes crônicos durante 20 meses através de xenodiagnósticos mensais.
Castro et al3 realizaram três xenodiagnósticos em um grupo de chagásicos durante dois anos e Schenone et al21 aplicaram xenodiagnósticos em um paciente durante 15 meses. O estudo mais longo da parasitemia ocorreu em um lapso de mais de 50 anos7 1718 mas, envolveu um único paciente. Em geral, são poucos os pacientes incluídos nos estudos longitudinais da parasitemia na doença de Chagas. Nesta oportunidade, apresentamos um estudo de 13 anos abrangendo grande número de chagásicos.

\section{MATERIAL E MÉTODOS}

Este trabalho foi realizado de 1976 a 1991, em 202 pacientes, do município de Mambaí, GO, que tinham as reações de fixação do complemento, hemaglutinação passiva e imunofluorescência indireta para infecção chagásica, feitas em dois laboratórios de referência.

Para avaliar a parasitemia de cada indivíduo, o xenodiagnóstico foi realizado em três etapas. Entre junho de 1976 e setembro de 1978, foram feitos três exames em todos os 202 indivíduos. Entre outubro de 1978 a 1984, foram realizados xenodiagnósticos em 48 chagásicos, 31 dos quais foram tratados especificamente nesse período. Finalmente, entre abril de 1988 e agosto de 1991, todos os 202 chagásicos fizeram um xenodiagnóstico. $O$ intervalo entre os exames foi variável. No primeiro xenodiagnóstico, de 126 chagásicos foi utilizado o Triatoma infestans, nos demais exames, o Dipetalogaster maximus.

Para a realização dos xenodiagnósticos, 40 triatomíneos foram distribuídos em quatro caixas de madeira, vedadas com tela de filó. Em cada caixa foram colocados 10 insetos do terceiro estágio de T. infestans, ou do primeiro estágio de $D$. maximus, e foram enviados à área endêmica. Os triatomíneos foram aplicados nas panturrilhas dos pacientes durante 30 minutos, após prévia limpeza da pele com algodão embebido em álcool. Após a aplicação, foi recomendado aos pacientes manterem a higiene da pele e evitar escoriações com as unhas. Os triatomíneos foram devolvidos ao laboratório em Brasília, sendo mantidos à temperatura ambiente, até a época do exame.

Os triatomíneos foram examinados em pool constituído de no máximo, cinco insetos. De cada caixa foram preparados dois pools. Quando o número de triatomíneos em uma caixa era inferior a dez, mas superior a cinco, um dos pools foi formado por cinco triatomíneos. Mesmo os triatomíneos mortos foram examinados, exceto quando estavam secos. Cada xenodiagnóstico teve um máximo de oito pools e os triatomíneos foram examinados, no mínimo, 30 dias após serem aplicados. A técnica usada consistiu na retirada do conteúdo intestinal de cinco insetos, depositado em lâmina, homogeneizado em uma gota de salina, coberto com lamínula de 32 por $24 \mathrm{~mm}$ e examinado com aumento de $100 x$ e, em caso de dúvida, com aumento de $400 x$ até a detecção do parasito. O exame foi considerado negativo quando em 10 a 15 minutos nenhum parasito era encontrado em toda a lâmina. $O$ resultado foi registrado em livro próprio, tendo cada xenodiagnóstico no máximo oito anotações, correspondentes às oito lâminas, o que possibilitou a semiquantificação do exame.

Para avaliar a parasitemia de cada indivíduo, utilizou-se a classificação arbitrária de Castro2 que leva em consideração o percentural de pools positivos em relação aos pools examinados. Assim, cada chagásico foi classificado como tendo baixa parasitemia (zero a 19,9\% pools positivos do total de pools examinados), média parasitemia (20 a 67,9\%) e alta parasitemia (68\% ou mais pools positivos do total de pools examinados), no conjunto dos três exames feitos em 1976/78 e no único exame realizado pelos 202 chagásicos em 1988/91.

Em 1980, foi realizada a borrifição com hexacloreto de benzeno, dos domicílios em todo o município, sendo em seguida realizados expurgos seletivos ${ }^{9}$, quando necessário, até aos dias atuais

\section{RESULTADOS}

As reações sorológicas dos 202 chagásicos, feitas em 1976, mostraram que 173 pacientes apresentaram todos os exames positivos, 25 tiveram entre três e cinco reações positivas e 
uma negativa, um chagásico teve quatro reações sorológicas positivas e cinco negativas e três chagásicos tiveram três reações positivas e três negativas. Estes quatro últimos apresentaram xenodiagnósticos positivos.

Os 202 indivíduos deste estudo representam aproximadamente um quinto dos chagásicos do município de Mambaí e estão distribuídos de acordo com o sexo e, com a idade à época do último xenodiagnóstico, sendo $124(61,4 \%)$ do sexo feminino e $78(38,6 \%)$ do sexo masculino. Predominaram os indivíduos na faixa etária de 40-49 anos, representando $23,3 \%$ da amostra. O percentual de indivíduos com 60 anos, ou mais, representou $12,4 \%$, sendo maior do que na faixa de 15 a 19 anos, que foi $6,9 \%$. A idade mínima e a máxima foram, respectivamente, 15 e 84 anos, sendo a média de idade igual a 41,4 anos.

Estudo geral evolutivo dos xenodiagnósticos. No período de 1976 a 1991, houve, em média, 13 anos de estudo. Nesse ínterim, em 154 chagásicos foram feitos quatro xenodiagnósticos e, em 48, entre cinco e nove exames. Cento e quarenta chagásicos apresentaram, ao menos, um xenodiagnóstico positivo. Na faixa etária de 15 a 19 anos, todos os indivíduos foram xenopositivos e a menor xenopositividade ocorreu na faixa etária de 40 a 49 anos (Tabela 1). Em relação ao sexo, houve maior índice de positividade no sexo masculino mas, sem significância estatística, $\chi^{2}=1,1622 ; p=0,2811$.

Tabela 1 - Percentagem de chagásicos crônicos xenopositivos de acordo com a idade* e sexo.

\begin{tabular}{|c|c|c|c|c|c|c|c|c|c|}
\hline \multirow{2}{*}{$\begin{array}{l}\text { Faixa etária } \\
\text { (anos) }\end{array}$} & \multicolumn{3}{|c|}{ Feminino } & \multicolumn{3}{|c|}{ Masculino } & \multicolumn{3}{|c|}{ Total } \\
\hline & pos & exam & $\%$ & pos & exam & $\%$ & pos & exam & $\%$ \\
\hline $15-19$ & 8 & 8 & 100,0 & 6 & 6 & 100,0 & 14 & 14 & 100,0 \\
\hline $20-29$ & 22 & 28 & 78,6 & 13 & 17 & 76,5 & 35 & 45 & 77,8 \\
\hline $30-39$ & 16 & 25 & 64,0 & 6 & 9 & 66,7 & 22 & 34 & 64,7 \\
\hline $40-49$ & 17 & 29 & 58,6 & 10 & 18 & 55,5 & 27 & 47 & 57,4 \\
\hline $50-59$ & 9 & 21 & 42,9 & 13 & 16 & 81,2 & 22 & 37 & 59,4 \\
\hline $60 \geq$ & 10 & 13 & 76,9 & 10 & 12 & 83,3 & 20 & 25 & 80,0 \\
\hline Total & 82 & 124 & $66,1^{* *}$ & 58 & 78 & $74,3^{\star *}$ & 140 & 202 & 69,4 \\
\hline
\end{tabular}

* Idade à época do último xenodiagnóstico; $\quad \chi^{2}=1,1622 \quad{ }^{* *} p=0,2811$

pos $=$ positivos, exam $=$ examinados

Sessenta e dois chagásicos apresentaram todos os xenodiagnósticos negativos e 23 foram positivos em todos os exames. Dos 934 xenodiagnósticos realizados, $362(38,8 \%)$ foram positivos.

Considerando a ordem cronológica dos exames, verifica-se que todos os 202 indivíduos realizaram os três primeiros e o último xenodiagnóstico. O número de indivíduos positivos nestes exames foi, respectivamente, 76 (37,6\%), 98 (48,5\%), 103 (51\%) e 61 (30,2\%) (Tabela 2 e Figura 1). Houve diferença estatisticamente significante entre os três primeiros e o último exame, $\chi^{2}=23,3763$ $p=0,00003$.

De outubro de 1978 a 1984, foram tratados 31 chagásicos com nifurtimox ou benznidazol e, a baixa positividade dos exames nesta época

Tabela 2 - Resultados dos xenodiagnósticos de 202 chagásicos crônicos, segundo a cronologia dos exames.

\begin{tabular}{|c|c|c|c|c|}
\hline \multirow[t]{2}{*}{ Período dos exames } & \multirow[t]{2}{*}{ Ordem dos exames } & \multirow[t]{2}{*}{ Número indivíduos examinados } & \multicolumn{2}{|c|}{ Indivíduos positivos } \\
\hline & & & $\mathrm{n}^{\circ} \mathrm{o}$ & $\%$ \\
\hline \multirow{3}{*}{$1976 / 78$} & $1^{0}$ & 202 & 76 & 37,6 ** \\
\hline & $2^{\circ}$ & 202 & 98 & $48,5^{* *}$ \\
\hline & $3^{\circ}$ & 202 & 103 & 51,0 ** \\
\hline \multirow{5}{*}{$1978 / 84^{*}$} & $4^{\circ}$ & 48 & 11 & 22,9 \\
\hline & $5^{0}$ & 37 & 9 & 24,3 \\
\hline & $6^{0}$ & 27 & 3 & 11,1 \\
\hline & $7^{0} \underline{0}$ & 13 & 1 & 7,7 \\
\hline & $8^{\circ}$ & 1 & 0 & - \\
\hline $1988 / 91$ & último & 202 & 61 & 30,2 ** \\
\hline Total & & 934 & 362 & 38,8 \\
\hline
\end{tabular}

* 31 foram tratados especificamente após realizar o terceiro xenodiagnóstico

${ }^{\star *} \chi^{2}=23,3763 p=0,00003$ 
deve-se, provavelmente, ao tratamento (Tabela 2).

O estudo feito em 1976/78 e repetido em 1988/91, nos 202 pacientes, revelou que houve queda da parasitemia. A Tabela 3 e a Figura 2 mostram que o percentual de pools positivos no primeiro, segundo, terceiro e último xenodiagnósticos foi, respectivamente, 15,2\% (201/1323), 20,9\% (325/1556), 20,8\% (321/1541) e $10,4 \%(164 / 1582)$. Excluíndo todos os xenodiagnósticos dos 31 pacientes tratados, obtêm-se respectivamente $13,9 \%(156 / 1120)$, $19 \%$ (267/1332), 20,4\% (267/1307) e 10,8\% (146/1345) pools positivos, números bastante idênticos aos anteriores, mostrando que o tratamento dos 31 pacientes não influiu no declínio da parasitemia dos 202 chagásicos. Ressalte-se que, dos 31 chagásicos tratados $9(29 \%)$ voltaram a apresentar parasitemia.

Estudo longitudinal dos chagásicos com alta parasitemia. Em trabalho anterior3, estudando 292 chagásicos crônicos, foi mostrado que 9,2\%,

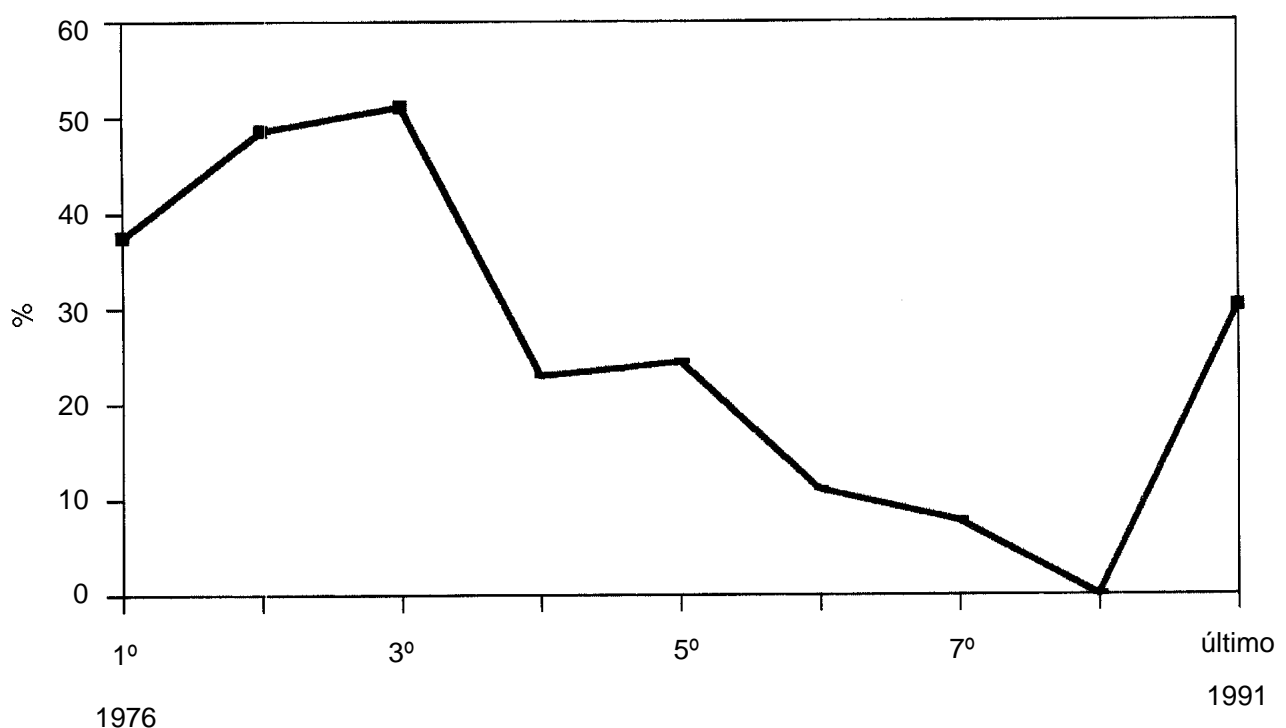

xenodiagnósticos

Figura 1 - Percentual de chagásicos xenopositivos no período médio de 13 anos.

Tabela 3 - Número de pessoas e de pools examinados e positivos em cada xenodiagnóstico.

\begin{tabular}{|c|c|c|c|c|}
\hline \multirow{2}{*}{ Ordem dos Xenos } & \multirow{2}{*}{ Número de pessoas } & \multicolumn{3}{|c|}{ Número de pools } \\
\hline & & exam & pos & $\%$ \\
\hline$\overline{10}$ & 202 & 1323 & 201 & $15,2^{* *}$ \\
\hline $2^{\circ}$ & 202 & 1556 & 325 & 20,9 ** \\
\hline $3^{\circ}$ & 202 & 1541 & 321 & 20,8 ** \\
\hline $4^{\underline{0 *}}$ & 48 & 367 & 36 & 9,8 \\
\hline $5^{0}$ & 37 & 273 & 23 & 8,4 \\
\hline $6^{\circ}$ & 27 & 202 & 3 & 1,5 \\
\hline $7^{\circ}$ & 13 & 91 & 3 & 3,3 \\
\hline $8^{\circ}$ & 1 & 8 & 0 & - \\
\hline UX & 202 & 1582 & 164 & 10,4 ** \\
\hline
\end{tabular}

${ }^{\star} 31$ foram tratados especificamente após realizar o terceiro xeno; ${ }^{* \star} p<0,00000001 ; \chi^{2}=85,6044$;

exam = examinados; pos = positivos; UX = último xeno. 


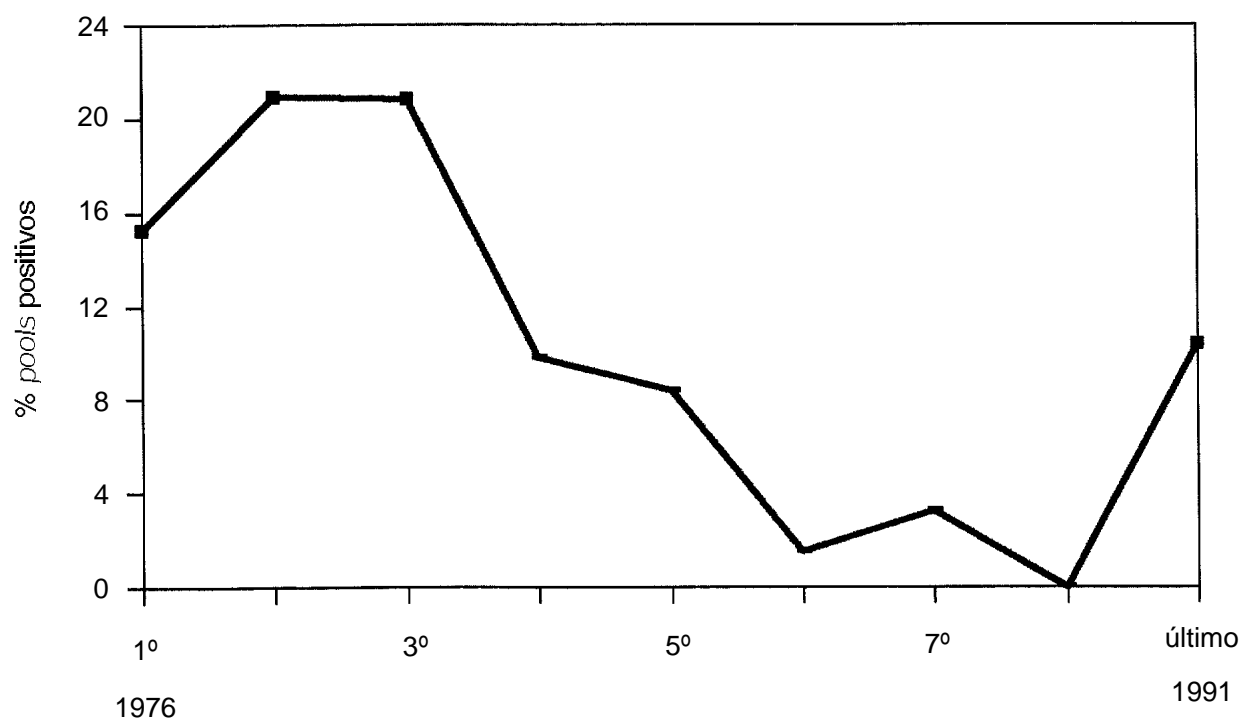

xenodiagnósticos

Figura 2 - Percentual de pools positivos de 202 chagásicos no período médio de 13 anos.

$23,3 \%$ e $67,5 \%$ dos pacientes tiveram alta, média e baixa parasitemia respectivamente. $O$ presente trabalho pretende mostrar a evolução da parasitemia de 202 daqueles chagásicos. Foram avaliados 19 (73\%) dos que tinham alta parasitemia e constatado que sete permaneceram com alta parasitemia, seis continuaram com parasitemia mas em níveis inferiores e, seis mostraram parasitemia negativa (Tabela 4).
Dos sete pacientes que continuaram com alta parasitemia, um foi tratado com nifurtimox durante 90 dias, teve breve período de negativação mas, recuperou a alta parasitemia.

Dos seis nos quais houve declínio da parasitemia, um foi tratado com nifurtimox durante 60 dias, negativou os exames temporariamente, mas voltou a apresentar parasitemia. Os outros seis que evoluíram com xenodiagnósticos

Tabela 4 - Evolução da parasitemia de 202 chagásicos crônicos, nos períodos de 1976/78 a 1988/91.

\begin{tabular}{lcrr} 
Parasitemia & Pacientes & \multicolumn{2}{c}{ Evolução da parasitemia em 1988/91 } \\
\cline { 2 - 4 } $1976 / 78$ & $n^{0}$ & alta & média \\
\hline Alta & 19 & 7 & 3 \\
Média & 42 & 1 & 11 \\
Baixa & 141 & 1 & 12 \\
\hline Total & 202 & 9 & 128 \\
\hline
\end{tabular}

negativos, um foi tratado com nifurtimox e dois com benznidazol.

Estudo longitudinal dos chagásicos com média parasitemia. Somente 42 indíviduos, que tinham média parasitemia em 1976/78, realizaram o último xenodiagnóstico em 1988/91. Um chagásico evoluiu de média para alta parasitemia, 11 continuaram com média parasitemia e 30 evoluíram para baixa parasitemia (Tabela 4). Dos 30 que evoluíram com baixa parasitemia, 18 apresentaram xenodiagnósticos negativos, dos quais sete foram tratados com benznidazol ou nifurtimox.

Estudo longitudinal dos chagásicos com baixa parasitemia. O comportamento de 141 chagásicos que tinham baixa parasitemia em 
1976/78, e que fizeram xenodiagnóstico em 1988/91, foi o seguinte: um evoluiu para alta parasitemia, 12 para média, e 128 continuaram com baixa parasitemia (Tabela 4), porém com percentual de pools positivos significativamente inferior ao exame feito em 1976/78.

Redistribuição dos chagásicos conforme a parasitemia de 1988/91. Os resultados dos xenodiagnósticos feitos em 1988/91 mostraram que 146 (72,3\%) chagásicos permaneceram com o mesmo nível da parasitemia inicial, $42(20,8 \%)$ baixaram o nível de parasitemia e 14 (6,9\%) elevaram-no.

A Tabela 4 mostra as modificações na parasitemia no período de $1988 / 91$, em relação a 1976/78. Dos 19 chagásicos com alta parasitemia em 1976/78, sete permaneceram com alta parasitemia, três passaram para a média e nove evoluíram com baixa parasitemia.
Dos 42 com média parasitemia, um evoluiu para alta parasitemia, 11 continuaram com média e 30 evoluiram com baixa parasitemia.

Dos 141 com baixa parasitemia, um evoluiu com alta parasitemia, 12 para a média e 128 continuaram com baixa parasitemia.

A evolução da parasitemia em 1988/91, mostra que nove chagásicos apresentaram alta parasitemia, sendo que um proveio da média e outro da baixa parasitemia. Dos 26 chagásicos com média parasitemia, três foram provenientes da alta e 12 foram procedentes da baixa parasitemia. Dos 167 com baixa parasitemia, nove tiveram origem entre os indivíduos da alta e 30 vieram dos individuos de média parasitemia (Tabela 4). Dos 167 chagásicos, apenas 26 apresentaram xenodiagnósticos positivos.

ATabela 5 também mostra, de outra maneira, as modificações nos níveis de parasitemia, nos

Tabela 5 - Nível da parasitemia de 202 chagásicos nos períodos de 1976/78 e 1988/91.

\begin{tabular}{lrrrrr}
\hline Parasitemia & \multicolumn{3}{c}{$1976 / 78$} & \multicolumn{2}{c}{$1988 / 91$} \\
\cline { 2 - 5 } & $\mathrm{n}^{0}$ & $\%$ & $\mathrm{n}^{\mathbf{0}}$ & $\%$ \\
\hline Alta & 19 & 9,4 & 9 & 4,4 \\
Média & 42 & 20,8 & 26 & 12,9 \\
Baixa & 141 & 69,8 & 167 & 82,7 \\
\hline Total & 202 & 100,0 & 202 & 100,0 \\
\hline
\end{tabular}

períodos acima mencionados. Deve ser ressaltado que seis pessoas só apresentaram xenodiagnóstico positivo após o terceiro exame, e uma somente após o sexto.

\section{DISCUSSÃO}

Os estudos longitudinais da parasitemia, propriamente ditos, foram iniciados na década de 70 por Cerisola et al. São passados mais de 20 anos e existem poucos trabalhos na literatura6 1415 , sobre o tema.

O xenodiagnóstico tem sido o procedimento de escolha para este propósito20. Todos os estudos longitudinais da parasitemia foram feitos com este método, apesar de sua baixa sensibilidade na doença de Chagas crônica. Mesmo assim, este procedimento deverá continuar sendo o preferido ainda por algum tempo, por ser mais eficiente e mais simples quando aplicado nas condições de trabalho na área rural. Quando repetido várias vezes, o xenodiagnóstico convencional pode causar sensibilização e reações sistêmicas em alguns pacientes 4 , daí a ênfase que tem sido dada, recentemente, ao xenodiagnóstico artificial 19.
Neste trabalho, avaliou-se a parasitemia dos pacientes por um período médio de 13 anos, que pode ser considerado longo, comparativamente aos estudos similares existentes.

Apesar do T. infestans ter sido eliminado gradualmente dos domicílios, a partir de 1980, quando foi executada a borrifação domiciliar em massa com hexacloreto de benzeno, seguida de expurgos seletivos com piretróides 91011 12, foi observado que sete chagásicos continuaram com alta parasitemia, 11 permaneceram com média parasitemia e 14 aumentaram o nível de parasitemia.

Dos 934 xenodiagnósticos realizados, 724 foram feitos antes da borrifação em massa e 210 no curso dos expurgos seletivos. Mesmo considerando que a queda da infestação triatomínica domiciliar foi gradual9, o fato de 32 $(15,8 \%)$ chagásicos manterem níveis 
relativamente altos ou elevarem a parasitemia, na ausência de triatomíneos nas residências, merece reflexão. Fica-se em dúvida quais são os fatores responsáveis pela manutenção prolongada de tais níveis de parasitemia, em indivíduos aparentemente imunocompetentes. Tudo indica que a parasitemia do chagásico depende mais da interação parasito hospedeiro, que de repetidas picadas de triatomíneos infectados.

A parasitemia persistente pode ser uma consequência do reservatório de $T$. cruzi na veia central da supra-renal23 devido a alta concentração local de corticóides. Segundo Teixeira et al22 alguns pacientes têm parasitismo intenso neste sítio que pode ser a fonte mantenedora da parasitemia em chagásicos imunocompetentes. Estes autores estudando 47 chagásicos com parasitismo da veia central da supra-renal encontraram em 6,3\% centenas de ninhos de amastigotas. Talvez os chagásicos com alta parasitemia ou com parasitemia persistente, sejam aqueles que possuem intenso parasitismo da veia central da supra-renal.

Entre os chagásicos que aumentaram a parasitemia durante o período deste estudo, um era alcoólatra crônico e um outro tinha pênfigo foliáceo e usava corticóide. Nestes dois casos, haveria alguma explicação para a alta parasitemia.

Talvez seja importante dosar o cortisol plasmático, para verificar se há alguma relação entre carga parasitária e nível hormonal. É possível que uma concentração elevada de cortisol, a nível da veia central da supra-renal, diminua o processo inflamatório em torno dos ninhos parasitários supra-renais 16 facilitando condições para que o parasitismo tecidual se dissemine sistemicamente.

Apesar de 32 chagásicos elevarem o nível ou permanecerem com parasitemia alta, o que ocorreu, em geral, foi o declínio da parasitemia que pode ser evidenciado pela redução do percentual de pools positivos, que passou de $20,9 \%$ no segundo exame para $10,4 \%$ no último xenodiagnóstico (Tabela 3 ).

O estudo longitudinal dos 202 chagásicos avaliado pelo número de pacientes xenopositivos e pelo número de pools positivos, nos três exames realizados, antes da borrifação domiciliar e no último xenodiagnóstico, mostra queda da parasitemia (Tabelas 2 e 3, Figuras 1 e 2). Podese imaginar que o declínio da parasitemia foi consequência da borrifação. Como não existem trabalhos mostrando que expurgo com inseticida ocasiona queda da parasitemia pelo T. cruzi, em população chagásica, não se pode responder a esta questão. Uma vez que 32 indivíduos permaneceram com parasitemia alta ou relativamente alta, durante o período de estudo, pode-se pensar que a eliminação dos triatomíneos das residências não influencia o declínio da parasitemia.

Também, não se pode atribuir a queda da parasitemia dos 202 chagásicos, ao tratamento específico de 31 pacientes, porque nove deles recuperaram a parasitemia posteriormente e, em segundo lugar, porque quando se exclui os exames dos 31 que foram tratados, o percentual de pools positivos continua idêntico

Houve 23 chagásicos neste estudo que apresentaram todos os xenodiagnosticos positivos. Considerando que a maioria provavelmente foi infectada na infância e na adolescência, e que 12 deles tinham entre 40 e 78 anos, pode-se supor que alguns permaneçam a vida inteira com parasitemia patente ao xenodiagnóstico.

Não se conhece a relação entre parasitismo tecidual e parasitemia. Não sabemos se um indivíduo com parasitismo intenso da veia central da supra-renal22 tem parasitemia elevada e vice-versa.

É sabido que o parasitismo tissular não se extingue e que, potencialmente, o T. cruzi está sempre multiplicando e destruindo células, possivelmente em decorrência do estado imune. É possível haver maior multiplicação em períodos de imunodeficiência celular.

Apesar do $T$. cruzi ter a capacidade de parasitar qualquer tecido, tal parasitismo segundo Barbosa e Andrade1, desperta pouca reação inflamatória nos tecidos, exceto no músculo cardíaco, onde os parasitos, quando presentes, estão associados com inflamação crônica difusa e progressiva.

E provável que o parasitismo tecidual permaneça no indivíduo para sempre. Isto pode ser constatado nos pacientes com insuficiência cardíaca e graves arritmias que, ao serem submetidos a transplante cardíaco e ao tratamento imunossupressor, reativam o parasitismo24. O parasitismo é encontrado também nos indivíduos chagásicos que falecem com insuficiência cardíaca 1 . Neste estudo, foi encontrada parasitemia em $80 \%$ dos chagásicos, com 60 anos ou mais, mostrando que o T. cruzi acompanha a longevidade do indivíduo. 
O encontro do $T$. cruzi na veia central da supra-renal revitalizou a teoria segundo a qual, a presença do parasito é importante na patogenia da doença e estaria sempre sendo semeado para outros sítios do organismo, especialmente em condições de depressão imunitária. A síndrome da imunodeficiência adquirida, o tratamento de manutenção dos transplantados renais e cardíacos e a quimioterapia das doenças malignas têm contribuído para reativar o $T$. cruzi latente, mostrando que este persiste no organismo e ressurge, especialmente, na pele e no coração transplantados 24 . Mas, mesmo no paciente imunocompetente, embora a parasitemia em geral diminua ao longo do tempo, ela pode ser demonstrada durante muitos anos e, em alguns pacientes, pode ter níveis elevados.

\section{REFERÊNCIAS BIBLIOGRÁFICAS}

1. Barbosa Jr AA, Andrade ZA. Identificação do Trypanosoma cruzi nos tecidos extracardiacos de portadores de miocardite crônica chagasica. Revista da Sociedade Brasileira de Medicina Tropical 17:123-126, 1984

2. Castro $\mathrm{C}$. Influência da parasitemia no quadro clínico da doença de Chagas. Revista de Patologia Tropical 9:73136, 1980.

3. Castro C, Alves MT, Macêdo V. Importância da repetição do xenodiagnóstico para avaliação da parasitemia na fase crônica da doença de Chagas. Revista da Sociedade Brasileira de Medicina Tropical 16:98-103, 1983.

4. Castro C, Macêdo V. Reações severas na realização do xenodiagnostico. Revista da Sociedade Brasileira de Medicina Tropical 22 (supl II):134, 1989.

5. Cerisola JA, Rohwedder R. Comportamiento de la parasitemia y el immunodiagnostico de la infeccion chagasica cronica. In: Simpósio Internacional sobre Enfermedad de Chagas. Sociedad Argentina de Parasitologia, Buenos Aires, Argentina, p 271-275, 1972

6. Cerisola JA, Rohwedder R, Segura EL, Del Prado CE, Alvarez M, De Martini GJW. El xenodiagnostico. Ministério de Bienestar Social, Buenos Aires, Argentina, p 84, 1974.

7. Chagas C. Nova Trypanosomiase humana. Estudos sobre a morfologia e o ciclo evolutivo do Schizotrypanum cruzi n. gen. $\mathrm{n}$ sp. ajente etiologico de nova entidade morbida no homem. Memórias do Instituto Oswaldo Cruz 1:159-218, 1909.

8. Coura JR. Evolutive pattern in Chagas' disease and the life span of Trypanosoma cruzi in human infection. American Trypanosomiasis Reseach, Belo Horizonte $p$. 378-386, 1875.

9. Garcia-Zapata MT, Marsden PD. Control of the transmission of Chagas' disease in Mambaí, Goiás, Brasil (1980-1988). The American Journal of Tropical Medicine and Hygiene 46:440-443, 1992.

10. Garcia-Zapata MT, Marsden PD, Virgens D, Penna R, Soares V, Brasil IA, Castro CN, Prata A, Macêdo V. O controle da transmissão da doença de Chagas em Mambaí, Goiás, Brasil (1982-1984). Revista da Sociedade Brasileira de Medicina Tropical 19:219-225, 1986.

11. Marsden PD. The control of Chagas' disease in Mambaí, Brazil. The initial phases. Infection Control 2:466470,1981
12. Marsden PD, Virgens D, Castro CN, Brasil IP, Ferreira R, Silveira AC, Matos CAS, Macêdo V, Prata A. The control of Chagas' disease transmission in Mambaí, Goiás Brasil (1980-1981). Revista da Sociedade Brasileira de Medicina Tropical 16:189-195, 1983.

13. Medina-Lopes MD, Macêdo V. Xenodiagnóstico no ciclo gravido-puerperal. Revista da Sociedade Brasileira de Medicina Tropical 25 (supl III):100-101, 1992.

14. Pereira JB. Doença de Chagas humana: estudo da infecção crônica, morbidade e mortalidade em Virgem da Lapa, MG, Brasil (1976-1996). Tese de Doutorado, Instituto Oswaldo Cruz, Rio de Janeiro, RJ, 1997.

15. Pereira JB, Willcox HP, Coura JR. Morbidade da doença de Chagas. III. Estudo longitudinal de seis anos, em Virgem da Lapa, MG, Brasil. Memórias do Instituto Oswaldo Cruz 80:63-71, 1985.

16. Rodrigues MLP, Reis MG, Franquini Júnior J, Castro ECC, Reis MA, Teixeira VPA. Inflamatory response against Trypanosoma cruzi parasited cells from adrenal vein and myocardium in chronic Chagas' disease. Revista da Sociedade Brasileira de Medicina Tropical 29: 507-508, 1996.

17. Salgado JA. O centenário de Carlos Chagas e a menina Berenice. Memórias do Instituto Oswaldo Cruz 75:193195, 1980.

18. Salgado JA, Garcez PN, Oliveira CA, Galizzi J. Revisão Clínica atual do primeiro caso humano de doença de Chagas. Revista do Instituto de Medicina Tropical de São Paulo 4:330-337, 1962.

19. Santos AH, Silva IG, Rassi A. Estudo comparativo entre o xenodiagnóstico natural e o artificial em chagásicos crônicos. Revista da Sociedade Brasileira de Medicina Tropical 28:367-373, 1995.

20. Schenone H, Contreras MC, Rojas A . Rendimiento del xenodiagnostico, segun el numero de cajas utilizadas en 1181 personas com infeccion chagasica cronica diagnosticada mediante la reaccion de hemaglutinacion indireta. Boletin Chileno de Parasitologia 46:58-61, 1991.

21. Schenone H, Rojo M, Rojas A, Concha L. Positividad diurna y nocturna del xenodiagnostico en un paciente com infeccion chagasica cronica de parasitemia permanente. Boletin Chileno de Parasitologia 32:63-66, 1977. 
22. Teixeira VPA, Fernandes PA, Brandão MC, Gobbi H, Reis MA, Almeida HO. Parasitismo incomum na veia central da supra-renal em chagásicos crônicos. Arquivos Brasileiros de Cardiologia 47:425-428, 1986.

23. Teixeira VPA, Reis MA, Araujo MBM, Silveira SA, Reis L, Almeida HO. Comparação do parasitismo da veia central da supra-renal com o de outros tecidos em chagásicos crônicos. Revista da Sociedade Brasileira de Medicina Troical 24:73-78, 1991.

24. Uip DE, Strabelli TMV, Belotti G, Bocchi EA, Stolf N, Pileggi $F$, Jatene AD, Amato Neto V. Evolução Clínica de pacientes com doença de Chagas submetidos a transplante cardíaco. Revista da Sociedade Brasileira de Medicina Tropical 20 (supl II):26, 1987. 